\title{
Altered respiratory microbiota composition and functionality associated with asthma early in life
}

\author{
Mohammad T. AL Bataineh ${ }^{1,2^{*}}$ (D), Rifat A. Hamoudi ${ }^{1,2,3}$, Nihar R. Dash ${ }^{1}$, Rakhee K. Ramakrishnan², \\ Mohamad A. Almasalmeh ${ }^{4}$, Hanan A. Sharif ${ }^{4}$, Mohamed S. Al-Hajjaj ${ }^{1,4}$ and Qutayba Hamid ${ }^{2,5}$
}

\begin{abstract}
Background: The microbiota of the respiratory tract has an important role in maintaining respiratory health. However, little is known on the respiratory microbiota in asthmatic patients among Middle Eastern populations. This study investigated the respiratory microbiota composition and functionality associated with asthma in Emirati subjects.

Methods: We performed 16S rRNA and ITS2-gene based microbial profiling of 40 expectorated sputum samples from adult and pediatric Emirati individuals averaging 52 and 7 years of age, respectively with or without asthma.

Results: We report bacterial difference belonging to Bacteroidetes, Firmicutes, Fusobacteria and Proteobacteria phyla between asthmatic and non-asthmatic controls. Similarly, fungal difference belonging to Ascomycota, Basidiomycota phyla and other unclassified fungi. Differential abundance testing among asthmatic individuals with relation to Asthma Control Test show a significant depletion of Penicillium aethiopicum and Alternaria spp., among poorly controlled asthmatics. Moreover, data suggest a significant expansion of Malassezia spp. and other unclassified fungi in the airways of those receiving steroids and leukotriene receptor antagonists' combination therapy, in contrast to those receiving steroids alone. Functional profiling from $16 \mathrm{~S}$ data showed marked differences between pediatric asthmatic and non-asthmatic controls, with pediatric asthmatic patients showing an increase in amino acid $\left(p\right.$-value $\left.<5.03 \times 10^{-7}\right)$, carbohydrate $\left(p\right.$-value $\left.<4.76 \times 10^{-7}\right)$, and fatty acid degradation ( $p$-value $<6.65 \times 10^{-7}$ ) pathways, whereas non-asthmatic controls are associated with increase in amino acid ( $p$-value $<8.34 \times 10^{-7}$ ), carbohydrate ( $p$-value $\left.<3.65 \times 10^{-7}\right)$, and fatty acid $\left(p\right.$-value $<2.18 \times 10^{-6}$ ) biosynthesis pathways in concordance with enterotype composition.

Conclusions: These differences provide an insight into respiratory microbiota composition in Emirati population and its possible role in the development of asthma early in life. This study provides important information that may eventually lead to the development of screening biomarkers to predict early asthma development and novel therapeutic approaches.
\end{abstract}

Keywords: Asthma, Microbiota, Fungal

\footnotetext{
* Correspondence: malbataineh@sharjah.ac.ae

${ }^{1}$ Clinical Sciences Department, College of Medicine, University of Sharjah, Post Code: 27272, Sharjah, United Arab Emirates

${ }^{2}$ Sharjah Institute for Medical Research, University of Sharjah, Sharjah, United Arab Emirates

Full list of author information is available at the end of the article
}

(c) The Author(s). 2020 Open Access This article is licensed under a Creative Commons Attribution 4.0 International License, which permits use, sharing, adaptation, distribution and reproduction in any medium or format, as long as you give appropriate credit to the original author(s) and the source, provide a link to the Creative Commons licence, and indicate if changes were made. The images or other third party material in this article are included in the article's Creative Commons licence, unless indicated otherwise in a credit line to the material. If material is not included in the article's Creative Commons licence and your intended use is not permitted by statutory regulation or exceeds the permitted use, you will need to obtain permission directly from the copyright holder. To view a copy of this licence, visit http://creativecommons.org/licenses/by/4.0/. The Creative Commons Public Domain Dedication waiver (http://creativecommons.org/publicdomain/zero/1.0/) applies to the data made available in this article, unless otherwise stated in a credit line to the data. 


\section{Background}

Specialized microbial communities composed of bacteria, viruses and fungi termed as "respiratory microbiota" inhabit the human respiratory tract spanning from nostrils to the alveoli. The respiratory microbiota is highly dynamic, constantly evolving and influenced by multiple factors including host and environment [1, 2]. The respiratory microbiota is crucial for the maintenance of respiratory physiology and homeostasis [3]. It plays a significant role in the maturation and maintenance of respiratory immune responses and provides resistance to respiratory pathogen colonization [4]. At the same time, it has also been implicated in the structural development and morphogenesis of the respiratory tract $[5,6]$ as well as the development of mucosal immunity $[7,8]$.

The composition of the respiratory microbiota is increasingly being characterized in humans. Bacterial families Dolosigranulum spp., Corynebacterium spp. [9], viral families Anelloviridae [10, 11], and fungi such as Aspergillus spp., Penicillium spp., Candida spp., Alternaria spp. [12] populate the upper respiratory tract. Similarly, the lower respiratory tract is dominantly colonized by bacteria including Haemophilus spp., Moraxella spp., Streptococcus spp., Staphylococcus spp., Firmicutes and Bacteroidetes, and fungal families such as Eremothecium, Systenostrema and Malassezia [1315]. Characterization of the microbial communities residing in spatial niches along the respiratory tract is essential to elucidate the complex roles played by the respiratory microbiota in the pathogenesis of respiratory diseases.

Recent advances in our understanding of the respiratory microbiota composition and its alteration in diversity or abundance called "dysbiosis" has been linked to several chronic respiratory diseases such as asthma, cystic fibrosis, bronchiectasis, and chronic obstructive pulmonary disease $[4,16]$. Asthma is a healthcare priority with significant social and economic impact on societies. Studies have linked nasopharyngeal colonization with Streptococcus spp., Moraxella spp., Haemophilus spp. Prevotella spp., and respiratory syncytial virus, early in life to the development of lower respiratory tract infections, consecutive atopic disease and future asthma [17-19]. In particular, early asymptomatic Streptococcus colonization strongly correlated with subsequent wheezing and asthma risk. An apparent disturbance in the characteristic composition of bacterial communities was observed in asthmatic airways when compared to their healthy counterparts $[20,21]$. The bronchial airway microbiota composition and diversity significantly correlated with the degree of bronchial hyperresponsiveness in suboptimally controlled asthmatics [22]. Higher abundance of Proteobacteria is frequently observed in asthma patients [20,22]. Bronchial microbiota also shows variations across the different endotypes of asthma [23]. Furthermore, the airway expansion of specific genera of gram-negative bacteria was noticed to induce corticosteroid resistance in asthmatic patients, indicating that the composition of airway microbiota may influence corticosteroid responsiveness in asthma [24].

The precise understanding of the composition of the respiratory microbiota, the mechanisms by which these microbes interact with host immunity, and their functional effects on the pathogenesis, exacerbations, and comorbidities of chronic respiratory diseases such as asthma is still unclear and need factual elucidation. Further, we are still unsure how the structural ligands and metabolites from these microbes interact with the host and alter the development and progression of respiratory diseases. Here, we investigated the composition, diversity and functionality of respiratory microbiome in a cohort of pediatric and adult asthmatic patients using sputum samples. We further, characterized the alterations in respiratory microbiota with age especially among the asthmatic population.

\section{Methods}

Hospital Ethics and Research Committee, a local research ethics committee at the University Hospital Sharjah, UAE approved the study protocol (REC number: UHS-HERC- 039 -09042018). All subjects participating in the study supplied informed consent.

In this case control study, we collected 40 spontaneous expectorated sputum samples from Emirati citizens. Spontaneous coughed up sputum (expectorated phlegm/ mucous) was the first preference of sample collection whenever possible in all subjects. The subjects were provided with a labelled sputum container. They were asked to take a deep breath, hold for a few seconds, exhale, repeat two or three times, and then cough: sputum was collected after a productive cough. Sputum induction was sometimes used in subjects especially in children when sputum could not be expectorated spontaneously. Sputum induction was performed under close medical supervision with nebulization and nasopharyngeal suction. Expectorated sputum was collected in a sterile container stored immediately into liquid nitrogen and then transferred to $-80^{\circ} \mathrm{C}$ for further analysis. DNA extraction, PCR, Sequencing and Sequence processing were analyzed as described in supplementary document.

Information on the characteristics of the subjects in this study such as age, gender, body mass index (BMI), ethnicity, and animal exposure among others has been provided (Table 1).

We have also assessed asthma symptoms by collecting a patient-completed Asthma Control Test (ACT) questionnaire as previously described, scoring $<16$ as uncontrolled, $16-19$ as partially controlled, and $20-25$ as controlled [25]. In summary, participants were residents of Sharjah, UAE. Asthma patients were defined as those individuals who had a current diagnosis of asthma, for example, by being on the outpatient asthma clinics 
Table 1 Demographics and Clinical Characteristics of Study Cohort

\begin{tabular}{lllll}
\hline Characteristics & $\begin{array}{l}\text { Adult Asthmatic } \\
(\boldsymbol{n}=\mathbf{1 0})\end{array}$ & $\begin{array}{l}\text { Adult Healthy } \\
(\boldsymbol{n}=\mathbf{1 0})\end{array}$ & $\begin{array}{l}\text { Pediatric Asthmatic } \\
(\boldsymbol{n}=\mathbf{1 1})\end{array}$ & $\begin{array}{l}\text { Pediatric Healthy } \\
(\boldsymbol{n}=\mathbf{9})\end{array}$ \\
\hline Age, years Mean (SD, range) & $63.9(12.7,39)$ & $40(10.6,32)$ & $6.7(4.1,12)$ & $8(3.1,8)$ \\
Ethnicity, ${ }^{a}$ Emirati (\%) & $100 \%$ & $100 \%$ & $100 \%$ & $100 \%$ \\
Gender (M\%, F \%) & $30: 70$ & $20: 80$ & 45.55 & $56: 44$ \\
BMl (Kg/m ${ }^{2}$ ) Mean (SD, range) & $31.5(6.7,21)$ & $25.3(4.7,17)$ & $21.6(7.2,20.7)$ & $18.9(5.2,13.9)$ \\
Animal exposure (yes \%) & $0 \%$ & $20 \%$ & $9 \%$ & $11 \%$ \\
Asthma Control Test Mean (SD, range) & $18(3.1,10)$ & N/A & $17.9(4.4,15)$ & N/A \\
\hline
\end{tabular}

N/A Not Applicable

${ }^{a}$ Native UAE citizens

registrar. Most of the asthma patients were on inhaled corticosteroids and scoring on average 18 per ACT. Controls were defined as individuals who on questioning did not report having current or previous asthma, eczema or hay fever. Use of antibiotics and/or prescribed probiotics in the past 3 months, any form of smoking, other respiratory diseases or infections among the participants were the exclusion criteria used in this study (Table 1).

\section{Functional profiling from $16 \mathrm{~S}$ data}

Gene family abundances from Kegg Orthology (KO) functional space were computed from 16S OTU data and GreenGenes taxonomic annotations with Phylogenetic Investigation of Communities by Reconstruction of Unobserved States 2 (PICRUSt2) [26]. Metabolic Modules were quantified from the PICRUSt KO abundance matrix with GOmixer R package [27]. Unsupervised Hierarchical Clustering analysis was carried out using pheatmap (https:// cran.rproject.org/web/packages/pheatmap/pheatmap.pdf) function and subtree analysis was carried out using cutree (https://stat.ethz.ch/R-manual/Rpatched/library/stats/html/ cutree.html) functions using in-house $\mathrm{R}$ script provided in supplementary document. Briefly, the cutree function allows the separation of the main tree into subtrees components using statistical methods [28]. The bar plots represent the relative difference between the average pathway abundance of pediatric asthmatics and healthy subjects of each pathway displaying ANOVA with $p$-value $<0.05$. This was carried out using in-house $\mathrm{R}$ script.

\section{Statistical analysis}

Alpha and beta diversity was calculated using Shannon and Bray-Curtis indices with significance of diversity differences was tested with an ANOVA. We excluded OTUs occurring with a count of less than 3 in at least $10 \%$ of the samples. Difference in bacterial microbiota composition was evaluated using PERMANOVA with smoking group as the main fixed factor. All statistical testing were performed in the R software environment.

\section{Results}

\section{Sequence curation and metrics}

Deep amplicon sequencing, combined with the principles of statistical ecology can be used to survey microbiome communities. The advent of massively parallel sequencing and increased computational power has enabled scientists to leverage big genomic data to answer biological questions. Here, we collected spontaneous expectorated sputum samples from 40 individuals in order to assess their microbiota composition. We sequenced 16Sv4 amplicons generated from DNA samples on a MiSeq and the resulting dataset had 5798 OTUs. An average of 42,657 quality-filtered reads were generated per sample. As demonstrated in the analytical flowchart (Fig. S1), sequencing quality for R1 and R2 was determined using FastQC 0.11.5, and visualized (Fig. S2). Similarly, we also sequenced ITS2 amplicons generated from DNA samples on the MiSeq. The resulting dataset had 4024 OTUs. An average of 41,635 quality-filtered reads were generated per sample. Sequencing quality for R1 and R2 was determined using FastQC 0.11.5, and visualized (Fig. S3). Next, we evaluated the taxonomic composition generated from these high-quality reads and classified them using Greengenes (v. 13_8) as the reference database for bacteria, and UNITE (v. 7.1) as the reference database for fungi. We aggregated OTUs into each taxonomic rank, and plotted the relative abundance of the most abundant ones. In the figure legends, the unfilled portion of the bar represents unclassified and lower-abundance taxa (Fig. S4).

\section{Respiratory microbiota of asthmatic subjects show lower levels of richness and complexity compared to healthy subjects}

To assess richness of the 40 samples along with the evenness of bacterial and fungal populations for different asthmatic and healthy groups, we calculated alpha diversity using Shannon index. Next, to evaluate microbiome composition similarity across samples, we used abundanceweighted sample pair-wise differences using the BrayCurtis dissimilarity. We observed a significant difference of bacterial and fungal populations among asthmatic groups compared to healthy groups (Fig. 1). Further, we 


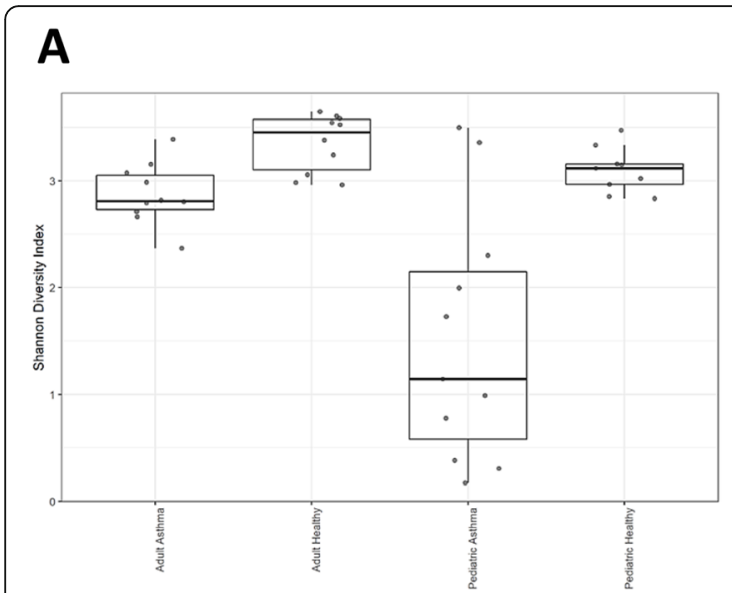

C

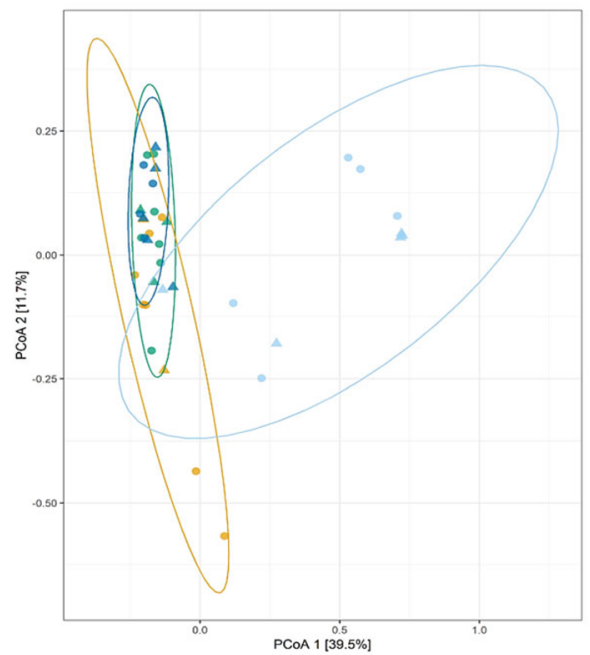

B

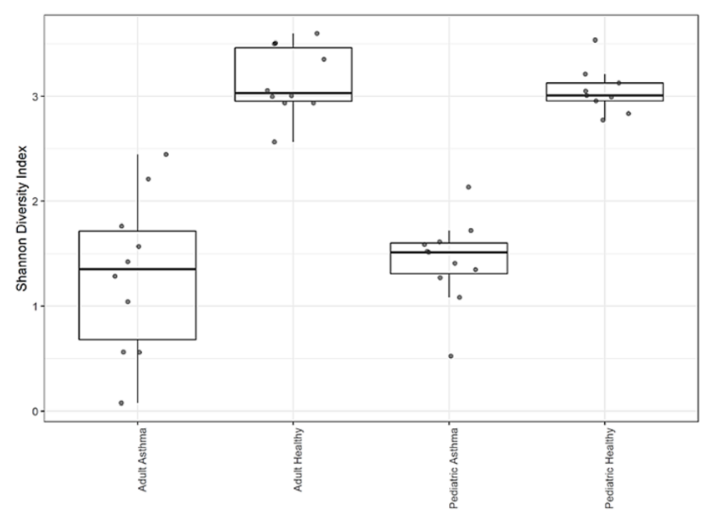

D

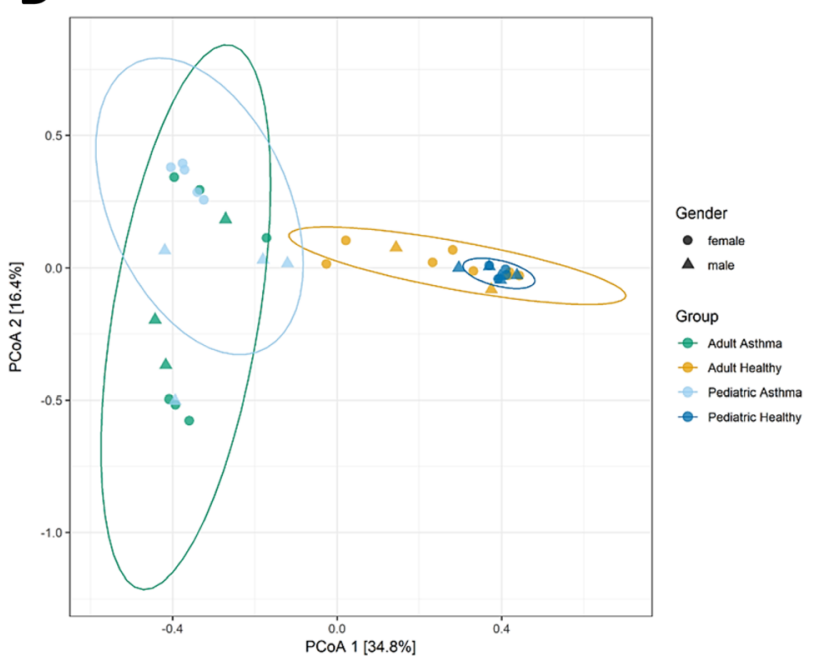

Fig. 1 Lung microbiota of asthmatic subjects show lower levels of richness and complexity compared to healthy subjects. Evaluation of the alpha- and beta- diversity in the 40 analyzed samples. Panel showing alpha-diversity (Shannon index) computed and illustrated for each sample. ANOVA test determined significant differences in the Shannon diversity index based on different groups for bacterial data at $p<0.002$ (a) and fungal data at $p<0.000$ (b). To obtain a graphical representation of microbiome composition similarity among samples and beta-diversity, we summarized OTU abundances into Bray-Curtis dissimilarities and performed a PCoA ordination. Permutational analysis of variance (adonis $R$ function, or Permanova) determined significant differences in beta-diversity among groups for bacterial data at $p<0.0009$ (c) and fungal data at $p<0.0001$ (d)

conducted a post-hoc pairwise test of different groups (asthmatic and healthy) and noted a uniformity in the expectorated sputum fungal composition between Adult Asthma vs. Pediatric Asthma groups (p.adjusted $>0.129$ ) (Table 2).

\section{Relative abundances of most abundant bacterial and fungal taxa in asthmatics}

In order to identify the important respiratory microbiota members that significantly differ between asthmatic and healthy groups as suggested in Fig. 1, we evaluated relative abundances of the five most abundant genus-level taxa within the four most abundant Phyla for bacteria and fungi (Fig. 2). We noted a significant bacterial difference belonging to Bacteroidetes, Firmicutes, Fusobacteria
Table 2 Beta diversity post-hoc pairwise test of different groups

\begin{tabular}{lll}
\hline Pairs & R2 & p.adjusted \\
\hline 16S (Bacterial) data & & \\
Adult Asthma vs Pediatric Asthma & 0.342 & 0.002 \\
Adult Asthma vs Adult Healthy & 0.118 & 0.023 \\
Pediatric Asthma vs Pediatric Healthy & 0.362 & 0.002 \\
ITS2 (Fungal) data & & \\
Adult Asthma vs Pediatric Asthma & 0.097 & $\mathbf{0 . 1 2 9}$ \\
Adult Asthma vs Adult Healthy & 0.318 & 0.002 \\
Pediatric Asthma vs Pediatric Healthy & 0.427 & 0.002 \\
\hline
\end{tabular}




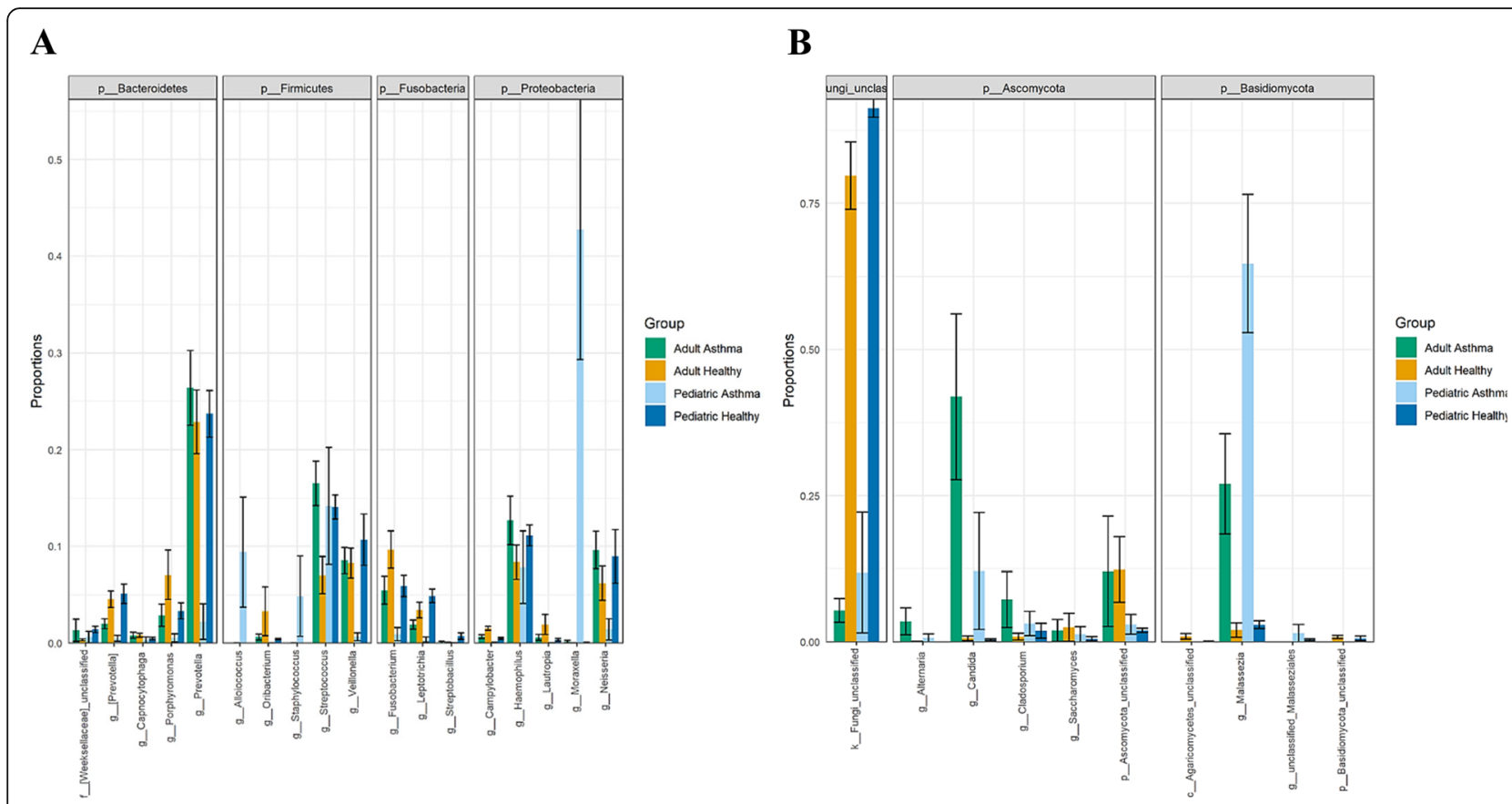

Fig. 2 Relative abundances of the most abundant taxa. The following plot illustrates the mean and standard error of the relative abundances of the 5 most abundant genus-level taxa within the 4 most abundant Phyla for bacteria (a) and fungi (b). The Genus-level plots are grouped according to Phylum along the $x$-axis. The groupings along the $y$-axis represent the column of metadata. The barplot colors represent different groups

and Proteobacteria phyla. Pediatric asthma group showed abundance in Streptococcus spp., Moraxella spp. among others. Moreover, we determined depletion of unclassified fungi in asthmatic groups and irrespective of age (Fig. 2b), we also noted significant abundance of Malassezia spp. and Candida spp. among others in asthmatic groups. A more detailed table summarizing genera with significant differences in relative abundances of bacterial and fungal overall diversity and prevalence among healthy and asthmatic groups, Kruskal-Wallace $P$-values as well as Benjamin-Hochberg corrected values are also reported (Table S1).

\section{Bacterial and fungal differential abundance among asthmatic individuals with relation to current medications and asthma control test (ACT)}

DESeq2 $\mathrm{R}$ package was used to identify differentially abundant taxa among medications used or ACT group variables. Differential abundance testing identified four bacterial OTUs, and two fungal OTUs that were differentially abundant with relation to ACT test result (partially controlled and controlled group vs. uncontrolled group) (Fig. 3a, c). ACT is a self-administered test to identify those with poorly controlled asthma [25, 29]. Accordingly, we show a significant depletion of Penicillium aethiopicum and Alternaria spp., with poorly controlled subjects (Fig. 3c). Also, we report differences of nine bacterial OTUs and three fungal OTUs when treated with steroids and leukotriene receptor antagonists (LTRA) in contrast to treatment with steroids alone (Fig. 3b, d). In particular, we reveal expansion of Malassezia spp. and other unclassified fungi (Fig. 3d) and unclassified genre belonging to Fusobacteria and Prevotella (Fig. 3b) in the airways of those receiving steroids and LTRA combination therapy.

\section{Functional profiling of asthmatic and healthy microbiota based on PICRUSt2 analyses of $16 \mathrm{~S}$ data}

We used PICRUSt analysis to predict functional contribution of the bacterial microbiota in the samples from $16 S$ OTU abundance data. Functional profiling showed significant overall differences in metabolic potential between asthmatics and healthy across age groups, especially among pediatric age groups (Fig. 4). Four hundred and ten significantly functional pathways were plotted in a heatmap showing a distinguished pattern of pathway enrichment in red or depletion in blue with relation to asthmatic and healthy groups (Fig. 4b). PICRUSt abundances permutational analysis of variance (adonis $R$ function, or Permanova) determined significant differences in beta-diversity among different groups at $p$ value $<0.0001$ (Fig. 4a). Moreover, derived from functional inference - PICRUSt2 data, average pathway abundance for pediatric asthmatic and healthy subjects of each pathway group was calculated using unsupervised hierarchical clustering followed by cutree to delineate 

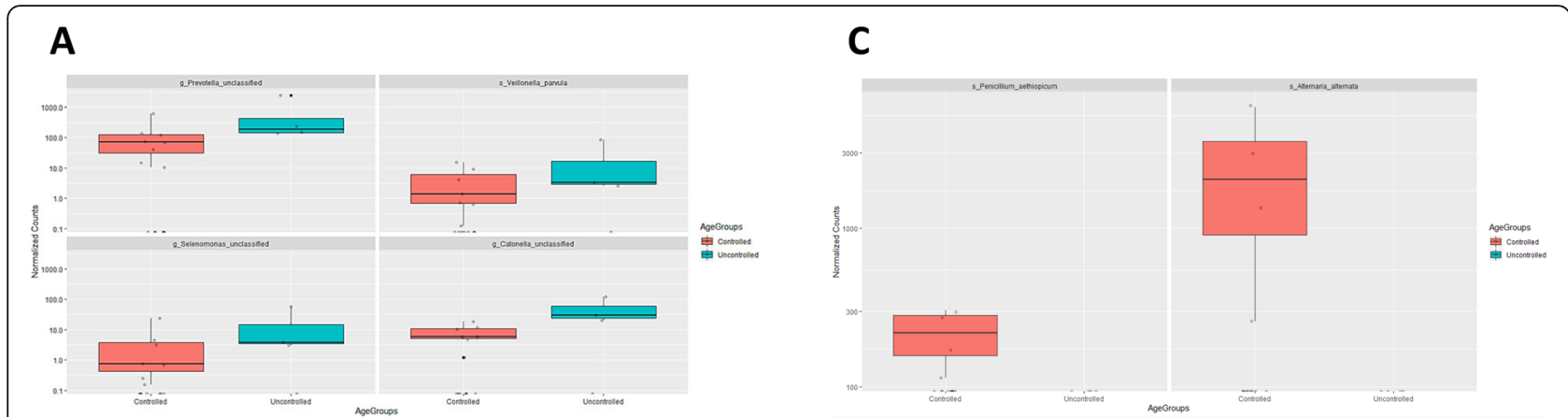

\section{B}

\section{D}
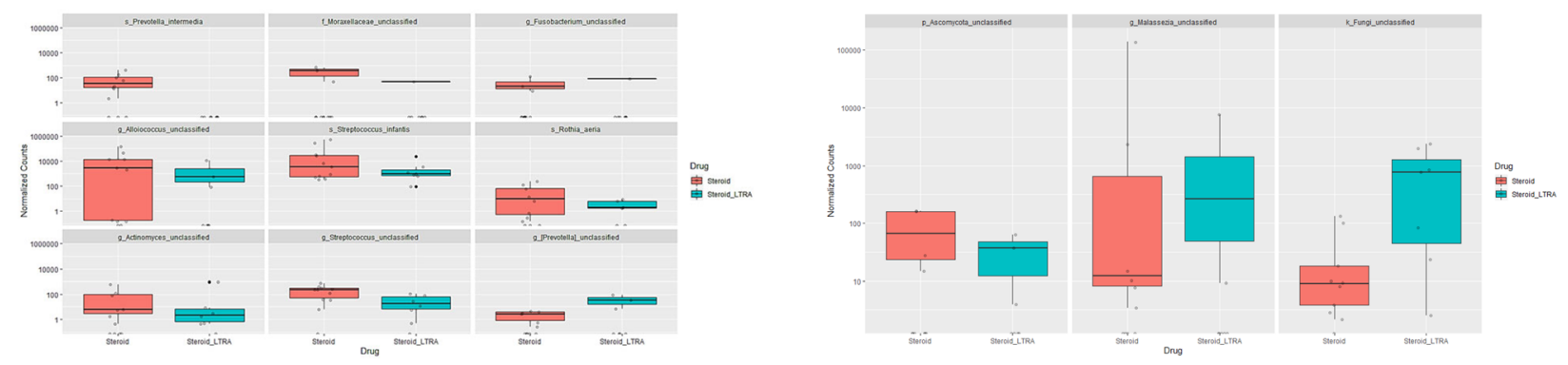

Fig. 3 Investigation of bacterial and fungal differential abundance among asthmatic individuals with relation to current medications and asthma control test (ACT). Differential abundance testing using DESeq2, R package to identify differentially abundant taxa among drug + ACT variables. The bar plot reports bacterial genera $(\mathbf{a}, \mathbf{b})$ and fungal genera $(\mathbf{c}, \mathbf{d})$ with significant abundance with relation to drug and ACT scores at a $p$-value $<0.05$ and the Log2Fc / Fold Change $>1.5$ or $<-1.5$ for both bacterial and fungal genera. Controlled and uncontrolled ACT scores as well as steroid and steroid + LTRA current treatment corresponding abundance are colored in red and blue, respectively

the healthy versus asthmatic pediatric patients. Cutree was used to extract the pediatric asthmatic patients from the mixed tree based on differentially expressed pathways. The region delineated using the cutree was then used to calculate the relative difference between the average abundance in healthy and asthmatic pediatric subjects for each pathway showing significant change between the pediatric asthma and healthy individuals based on ANOVA $p<0.05$ cutoff threshold for each pathway. We identified ninety pathways, in which pediatric asthmatic group show significant depletion in metabolic pathways implicated in amino acid, carbohydrate, and fatty acid biosynthesis, in contrast to significant enrichment of metabolic pathways involved in amino acid, carbohydrate, and fatty acid degradation in comparison to healthy pediatric group (Fig. 4c).

\section{Discussion}

In this study, we explored the composition and functional contribution of respiratory microbiota in asthmatics and their possible role with relation to ACT scores and asthma medication in adult and pediatric age groups. First, we observed that respiratory microbiota of asthmatic subjects showed lower levels of richness and complexity compared to healthy subjects. Consistent with our findings, previous reports have suggested a strong effect for asthma on lung microbiota composition
[30, 31]; however, very little has been reported on the role of fungal lung microbiota (mycobiota) in asthma. Here, we conducted a post-hoc pairwise test of different groups (asthmatic and healthy) and noted an intriguing uniformity in the expectorated sputum fungal composition between adult and pediatric asthma groups (p.adjusted $>0.129$ ) (Table 2). This may suggest an important role for lung mycobiota in driving these compositional changes and their contribution to asthma pathogenesis in early life that persist with age. Indeed, colonization patterns in infancy seem to be a major determinant of respiratory disease later in life [4]. Perhaps fungal presence induces changes in the lung micro-environmental conditions that subsequently drives this compositional shift among bacterial communities to compensate. For example, enrichment of Moraxella species in asthmatic airways was found to interact negatively with multiple bacterial communities but positively with fungal communities suggesting complex interactions between the bacterial and fungal communities that may contribute to asthma pathogenesis [32] That said, it is important to note that limited fungal genome database may also explain the identified similarity between adult and pediatric groups. For example, recent check (July, 2020) of the NCBI Genome database https://www.ncbi.nlm. nih.gov/genome/browse\#!/eukaryotes/fungi shown 6570 complete fungal genomes compared with $>252,000$ 


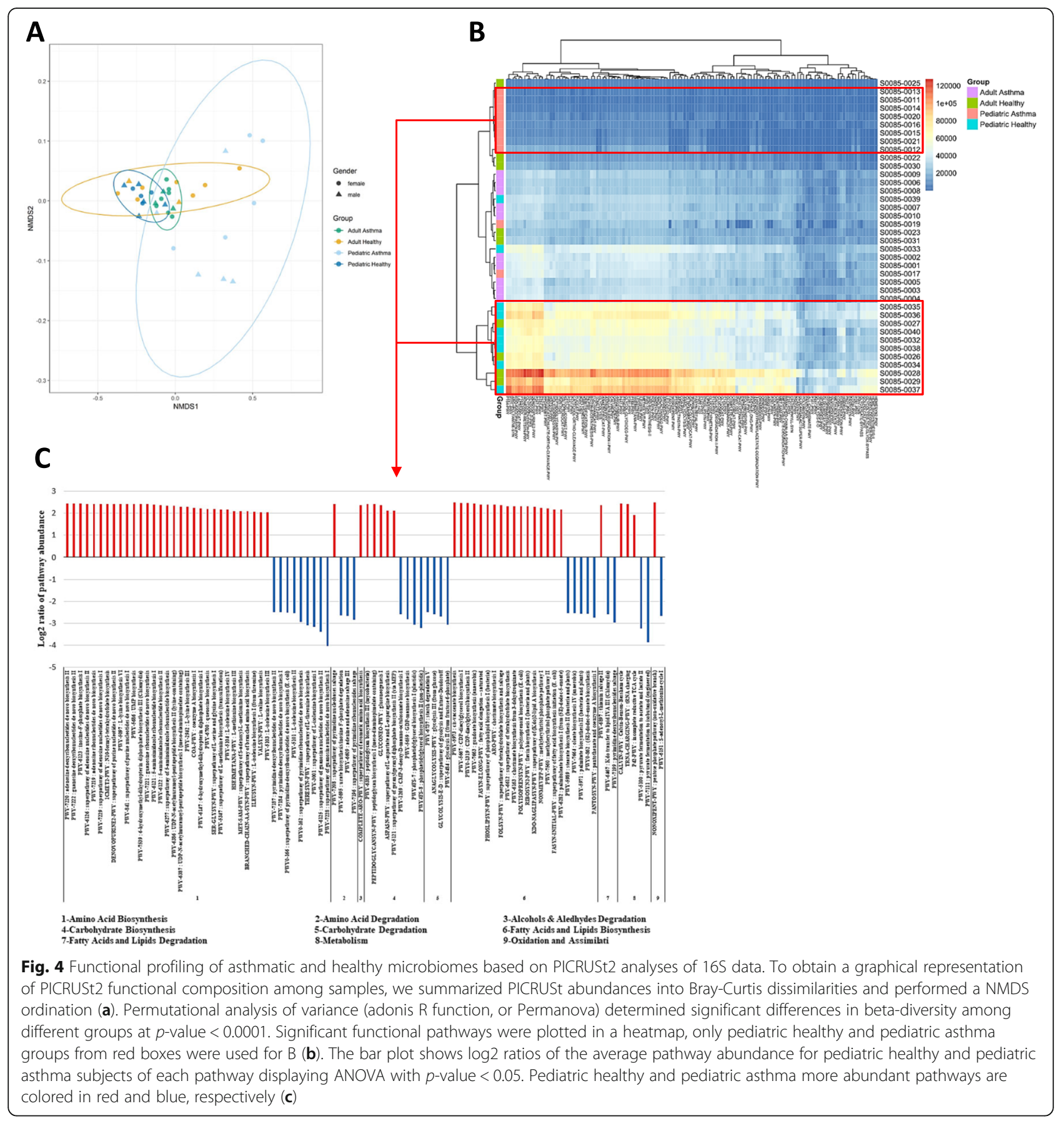

complete bacterial genomes. Altogether, these observations are interesting to identify those microbial populations that are more abundant in asthma and to further understand their relevance to asthma pathogenesis and host immune interactions. Next, we identified significant relative abundances of Bacteroidetes, Firmicutes, Fusobacteria and Proteobacteria phyla in asthmatics (Fig. 2). Further and consistent with previous study, we noted significant abundance of Malassezia spp. and Candida spp. in asthmatic groups [15].
We also showed abundance in Streptococcus spp., Moraxella spp. among other fungi in Pediatric Asthma group, and consistent with a previous study that links nasopharyngeal colonization with these species during early life to the development of lower respiratory tract infections, consecutive atopic disease and future asthma [17]. Moreover, we unraveled for the first time a staggering depletion of unclassified fungi in asthmatic groups and irrespective of age (Fig. 2b). These fungi, as eukaryotes, probably 
contribute through unique metabolic pathways to microbial equilibrium and host interactions to protect against asthma development.

Third, differential abundance testing with relation to asthmatic medications used or ACT score variables unveiled a significant depletion of Penicillium aethiopicum and Alternaria spp., in poorly controlled subjects (low ACT scores) (Fig. 3c). Previous studies indicated that indoor exposure to Penicillium spp., and Alternaria spp., is associated with active asthma symptoms [33-35]. Further, the richness and composition of respiratory microbiota of asthmatic patients were found to be significantly altered by inhaled and oral corticosteroid use [22, 36, 37]. On the other hand, the airway microbiome can also influence the corticosteroid responsiveness in asthma. A study by Goleva et al., noted expansion of the pathogenic Haemophilus parainfluenzae in corticosteroid-resistant asthma, and stimulation of bronchoalveolar lavage (BAL) macrophages with $H$. parainfluenzae promoted the activation of MAPK pathway and subsequent inhibition of corticosteroid responses, in contrast to commensal Prevotella melaninogenica [24]. However, little is known about the leukotriene receptor antagonists (LTRA) correlation with microbiota in asthma. Here, we report for the first-time differences of nine bacterial OTUs and three fungal OTUs when treated with steroids and LTRA in contrast to treatment with steroids alone (Fig. 3b, d). Interestingly, we demonstrate expansion of Malassezia spp., unclassified fungi, and genre belonging to unclassified Fusobacteria and unclassified Prevotella in the airways of those receiving steroids and LTRA combination therapy. These data can be useful to provide screening biomarkers to predict responsiveness to asthma management.

Lastly, utilizing PICRUSt analysis of the bacterial microbiota we revealed significant overall differences in metabolic potential between asthmatics and healthy across age groups, especially among pediatric age groups (Fig. 4). These differences provide an insight into respiratory microbiota fluctuations in Emirati population and its possible role in asthma pathogenesis. Moreover, using unsupervised hierarchical clustering followed by cutree to delineate the healthy versus asthmatic pediatric patients we identified ninety pathways, in which pediatric asthmatic group show significant depletion in metabolic pathways implicated in amino acid, carbohydrate, and fatty acid biosynthesis, in contrast to significant enrichment of metabolic pathways involved in amino acid, carbohydrate, and fatty acid degradation in comparison to healthy pediatric group (Fig. 4c). Maintenance of metabolic homeostasis, such as regulation of glucose uptake, amino acid acquisition and lipid synthesis are tightly governed by catabolic and anabolic events that ultimately dictates cell growth and proliferation, especially early in life [38]. The compromised balance between the two, as observed in our study, may contribute to disease pathogenesis within the lung milieu. For instance, elevation in multiple catabolic biomarkers is an indicator of accelerated decline of muscle strength [39]. A recent report sheds light on the cross talk between airway microbiota and host airway cells in signaling anabolic and catabolic remodeling of the transplanted lung [40]. Therefore, the increased anabolic ability among asthmatic group may ultimately enhance airway remodeling and inflammation, hallmark features of asthma. For example, among these pathways, we observed significant depletion of inosine degradation (PWY-5695) ( $p$-value < $\left.3.15 \times 10^{-7}\right)$, adenosine salvage (PWY-6609) ( $p$-value $\left.<5.48 \times 10^{-7}\right)$, and pyrimidine salvage (PWY-7196) $(p$ value $<6.18 \times 10^{-7}$ ) (Fig. $4 \mathrm{c}$ ) in pediatric asthmatics. Previous studies reports an important role for these purine metabolites in modulating lung inflammation $[41,42]$. Here, we observed significant depletion of the gram-positive Lactobacillus among pediatric asthmatic groups (P. adj <0.04) (Table S1). Interestingly, another study suggested that remodeling microbiota with Lactobacillus prolonged mice survival and reduced inflammation in the context of regulatory $\mathrm{T}$ cell dysfunction by restoring levels of inosine [43]. Moreover, other studies observed increased aerobic glycolysis and lactate production in asthma, which in turn promotes asthma development by $\mathrm{T}$ cell activation $[44,45]$. Here however, we show a significant depletion of glycolysis pathways (ANAGLYCOLYSISPWY, GLYCOLYSIS-E-D, PWY-5484) ( $p$-values < $3.13 \times 10^{-7},<7.89 \times 10^{-7},<1.96 \times 10^{-7}$ respectively) (Fig. 4c) in pediatric asthmatic group despite the reported increased relative abundance of lactic acid producing bacteria in this group, such as Streptococcus and Enterococcus [46] (P. adj <0.04, $<0.05$ respectively) (Table S1). Perhaps other major members of lung microbiota, such as Moraxella (\% absolute $42.75 \%$, P. adj < 0.00 ) (Table S1) in pediatric asthmatic group, in part explains the observed depletion of glycolysis pathways as previously reported to primarily drive gluconeogenesis [47]. Overall, these findings highlight the importance of respiratory microbiota in determining the airway microenvironment and thereby, influencing lung function.

Limitations of this study include that we used a small number of samples, which needs further validation on a larger cohort across populations with different environmental conditions and genetic background. We also used spontaneous expectorated sampling method that carry a high risk of cross-contamination of the lower respiratory tract microbiota with upper 
respiratory tract microbiota. Distinguishing between the two is difficult due to the anatomical link between both niches. That said, studies suggested that lung microbiota mostly resembles the upper respiratory tract microbiota [13, 48]. Fortunately, our expectorated sputum sampling is consistent with previous microbiome studies of bronchial sampling with high proportion of bacteria belonging to Bacteroidetes, Firmicutes, Fusobacteria and Proteobacteria phyla [21, 49]. Further, similar to our observation in pediatric asthmatics, a study noted that bacterial colonization of neonatal airways at 1 month of age was observed to predominate with pathogenic Streptococcus pneumoniae, Haemophilus influenzae or Moraxella catarrhalis, which in turn increased their risk and severity of wheeze as well as increased the total IgE and blood eosinophil counts by the age of 5 years, leading to their diagnosis of clinical asthma by age 5 and also implying their role in the development and progression of asthma [49]. Whereas another study by Huang et al., noted relative abundance of the airway microbiota belonging to other phylotypes, including members of Comamonadaceae, Sphingomonadaceae, Oxalobacteraceae bacterial families, correlated with the degree of bronchial hyperresponsiveness in patients with sub-optimally controlled asthma [22].

In conclusion, we identified respiratory bacterial and fungal species associated with asthmatic patients and shed more light on the functional impact of respiratory microbiota on asthma pathogenesis in correlation with management indices such as ACT test. Host and environmental factors dictates the respiratory microbiota makeup, which in turn will further shift the balance toward more inflammatory or protective role. These inflammatory responses govern the interplay between respiratory colonization and asthma development in a bidirectional manner that can be further complicated by asthma medications such as corticosteroids [4, 22, 24, 37]. Therefore, it is difficult to predict the potential contribution of the specific members of the airway microbiota towards asthma pathogenesis, underscoring the need for more functional studies and uniform sampling processes. We believe results from this study will further enhance our understanding of the composition and functionality of respiratory microbiota in asthmatic patients that can influence the potential manipulation of the microbiome as a therapeutic strategy for chronic respiratory diseases such as asthma. We hope that future work will provide a platform for better understanding of asthma pathophysiology. Most likely, these data can be useful to provide screening biomarkers to predict early asthma development and better management.

\section{Supplementary information}

Supplementary information accompanies this paper at https://doi.org/10. 1186/s12879-020-05427-3.

Additional file 1: Supplemental Materials and Methods.

Additional file 2: Figure. S1. Analytical flowchart outlining data curation and metrics used for analysis. Figure. S2. Bacterial sequence curation and analysis. We sequenced 16Sv4 amplicons generated from DNA samples on a MiSeq. MiSeq-generated Fastq files were qualityfiltered and clustered into $97 \%$ similarity operational taxonomic units (OTUs) using the mothur software package [http://www.mothur.org]. The per-base raw Q30 (Phred33) scores of the forward sequencing read are summarized (A). Sequencing quality for R1 and R2 was determined using FastQC 0.11.5, the per-sequence averaged raw Q30 (Phred33) scores of the forward sequencing read are summarized (B). Figure. S3. Fungal sequence curation and analysis. We sequenced ITS2 amplicons generated from DNA samples on a MiSeq. MiSeq-generated Fastq files were qualityfiltered and clustered into 97\% similarity operational taxonomic units (OTUs) using the mothur software package [http://www.mothur.org]. The per-base raw Q30 (Phred33) scores of the forward sequencing read are summarized (A). Sequencing quality for R1 and R2 was determined using FastQC 0.11.5, the per-sequence averaged raw Q30 (Phred33) scores of the forward sequencing read are summarized (B). Figure. S4. Aggregated taxonomic composition. High quality reads were classified using Greengenes v. 13_8 as the reference database for bactria and UNITE (v. 7.1) as the reference database for fungi. We aggregated OTUs into each taxonomic rank, the aggregated taxa were visualized at each taxanomic rank using taxanomic bar plots and plotted the relative abundance of the most abundant ones for bacteria (A) and Fungi (B). The unfilled portion of the bar plots represent lower-abundance taxa.

Additional file 3: Table S1. Summary of Bacterial and Fungal diversity and Prevalence Among Healthy and Asthmatic Groups Using KruskalWallace P-values and Benjamin-Hochberg Corrected Values.

\section{Abbreviations}

ACT: Asthma Control Test; ANOVA: Analysis of variance; BMI: Body mass index; KO: Kegg Orthology; OUT: Operational Taxonomic Unit;

PICRUSt2: Phylogenetic Investigation of Communities by Reconstruction of Unobserved States 2; PERMANOVA: Permutational analysis of variance

\section{Acknowledgments}

Sequencing data provided by microbiome insights, Canada.

Financial/nonfinancial disclosures

None declared.

\section{Authors' contributions}

Conception and design: MTA, MSA, NRD. Acquisition of data: MSA, MAA, HAS, RKR. Processing of specimens and generation of data: MTA, RAH, RKR, $\mathrm{QH}$. Analysis and interpretation of data: MTA, RAH, RKR, QH, NRD. Drafting or revising of manuscript: MTA, RAH, NRD, RKR, MAA, HAS, MSA, QH. Final approval of manuscript: MTA, RAH, NRD, RKR, MAA, HAS, MSA, QH. MTA has access to all study data and takes responsibility for the data integrity and accuracy. All authors have read and approved the manuscript.

\section{Funding}

The authors were supported by several UAE funding agencies; MTA funded by University of Sharjah (Grant code: 1901090253). RAH is funded by the Sharjah Research Academy (Grant code: MED001), University of Sharjah (Grant code: 1901090254) and the Al-Jalila Foundation (Grant code: AJF201741). The funding bodies had no role in the design of the study and collection, analysis, and interpretation of data and in writing the manuscript.

\section{Availability of data and materials}

The data that support the findings of this study are available on request from the corresponding author. The data are not publicly available due to privacy or ethical restrictions. 


\section{Ethics approval and consent to participate}

Hospital Ethics and Research Committee, a local research ethics committee at University Hospital Sharjah, UAE approved the study protocol (REC number: UHS-HERC- 039 -09042018). All subjects participated in the study supplied informed written consent.

\section{Consent for publication}

Not applicable.

\section{Competing interests}

The authors declare that they have no competing interests.

\section{Author details}

${ }^{1}$ Clinical Sciences Department, College of Medicine, University of Sharjah, Post Code: 27272, Sharjah, United Arab Emirates. ${ }^{2}$ Sharjah Institute for Medical Research, University of Sharjah, Sharjah, United Arab Emirates. ${ }^{3}$ Division of Surgery and Interventional Science, University College London, London, UK. ${ }^{4}$ University Hospital Sharjah, Sharjah, United Arab Emirates. ${ }^{5}$ Meakins-Christie Laboratories, McGill University, Montreal, QC, Canada.

Received: 29 April 2020 Accepted: 16 September 2020

Published online: 22 September 2020

\section{References}

1. Teo SM, Tang HHF, Mok D, Judd LM, Watts SC, Pham K, Holt BJ, Kusel M, Serralha M, Troy N, et al. Airway microbiota dynamics uncover a critical window for interplay of pathogenic Bacteria and allergy in childhood respiratory disease. Cell Host Microbe. 2018;24(3):341-52 e345.

2. Nguyen LD, Viscogliosi $E$, Delhaes $L$. The lung mycobiome: an emerging field of the human respiratory microbiome. Front Microbiol. 2015;6:89.

3. Mathieu E, Escribano-Vazquez U, Descamps D, Cherbuy C, Langella P, Riffault S, Remot A, Thomas M. Paradigms of lung microbiota functions in health and disease, particularly, in asthma. Front Physiol. 2018;9:1168.

4. Man WH, de Steenhuijsen Piters WA, Bogaert D. The microbiota of the respiratory tract: gatekeeper to respiratory health. Nat Rev Microbiol. 2017; 15(5):259-70.

5. Yun Y, Srinivas G, Kuenzel S, Linnenbrink M, Alnahas S, Bruce KD, Steinhoff $U$, Baines JF, Schaible UE. Environmentally determined differences in the murine lung microbiota and their relation to alveolar architecture. PLoS One. 2014;9(12):e113466.

6. Wostmann BS. The germfree animal in nutritional studies. Annu Rev Nutr. 1981;1:257-79.

7. Olszak T, An D, Zeissig S, Vera MP, Richter J, Franke A, Glickman JN, Siebert R, Baron RM, Kasper DL, et al. Microbial exposure during early life has persistent effects on natural killer T cell function. Science. 2012;336(6080): 489-93.

8. Gollwitzer ES, Saglani S, Trompette A, Yadava K, Sherburn R, McCoy KD, Nicod LP, Lloyd CM, Marsland BJ. Lung microbiota promotes tolerance to allergens in neonates via PD-L1. Nat Med. 2014;20(6):642-7.

9. Pettigrew MM, Laufer AS, Gent JF, Kong Y, Fennie KP, Metlay JP. Upper respiratory tract microbial communities, acute otitis media pathogens, and antibiotic use in healthy and sick children. Appl Environ Microbiol. 2012; 78(17):6262-70.

10. Wylie KM, Mihindukulasuriya KA, Sodergren E, Weinstock GM, Storch GA Sequence analysis of the human virome in febrile and afebrile children. PLoS One. 2012;7(6):e27735.

11. Wang Y, Zhu N, Li Y, Lu R, Wang H, Liu G, Zou X, Xie Z, Tan W. Metagenomic analysis of viral genetic diversity in respiratory samples from children with severe acute respiratory infection in China. Clin Microbiol Infect. 2016;22(5):458 e451-9.

12. Charlson ES, Diamond JM, Bittinger K, Fitzgerald AS, Yadav A, Haas AR, Bushman FD, Collman RG. Lung-enriched organisms and aberrant bacterial and fungal respiratory microbiota after lung transplant. Am J Respir Crit Care Med. 2012;186(6):536-45.

13. Marsh RL, Kaestli M, Chang AB, Binks MJ, Pope CE, Hoffman LR, SmithVaughan HC. The microbiota in bronchoalveolar lavage from young children with chronic lung disease includes taxa present in both the oropharynx and nasopharynx. Microbiome. 2016;4(1):37.

14. Segal LN, Alekseyenko AV, Clemente JC, Kulkarni R, Wu B, Gao Z, Chen H, Berger Kl, Goldring RM, Rom WN, et al. Enrichment of lung microbiome with supraglottic taxa is associated with increased pulmonary inflammation. Microbiome. 2013;1(1):19.

15. van Woerden HC, Gregory C, Brown R, Marchesi JR, Hoogendoorn B, Matthews IP. Differences in fungi present in induced sputum samples from asthma patients and non-atopic controls: a community based case control study. BMC Infect Dis. 2013;13:69.

16. Dumas A, Bernard L, Poquet $Y$, Lugo-Villarino G, Neyrolles O. The role of the lung microbiota and the gut-lung axis in respiratory infectious diseases. Cell Microbiol. 2018;20(12):e12966.

17. Teo SM, Mok D, Pham K, Kusel M, Serralha M, Troy N, Holt BJ, Hales BJ, Walker ML, Hollams E, et al. The infant nasopharyngeal microbiome impacts severity of lower respiratory infection and risk of asthma development. Cell Host Microbe. 2015;17(5):704-15.

18. Krishnamoorthy N, Khare A, Oriss TB, Raundhal M, Morse C, Yarlagadda M, Wenzel SE, Moore ML, Peebles RS Jr, Ray A, et al. Early infection with respiratory syncytial virus impairs regulatory $T$ cell function and increases susceptibility to allergic asthma. Nat Med. 2012;18(10):1525-30.

19. Larsen JM, Musavian HS, Butt TM, Ingvorsen C, Thysen AH, Brix S. Chronic obstructive pulmonary disease and asthma-associated Proteobacteria, but not commensal Prevotella spp., promote toll-like receptor 2-independent lung inflammation and pathology. Immunology. 2015;144(2):333-42.

20. Hilty M, Burke C, Pedro H, Cardenas P, Bush A, Bossley C, Davies J, Ervine A, Poulter L, Pachter L, et al. Disordered microbial communities in asthmatic airways. PLoS One. 2010;5(1):e8578.

21. Marri PR, Stern DA, Wright AL, Billheimer D, Martinez FD. Asthma-associated differences in microbial composition of induced sputum. J Allergy Clin Immunol. 2013;131(2):346-52 e341-343.

22. Huang YJ, Nelson CE, Brodie EL, Desantis TZ, Baek MS, Liu J, Woyke T, Allgaier M, Bristow J, Wiener-Kronish JP, et al. Airway microbiota and bronchial hyperresponsiveness in patients with suboptimally controlled asthma. J Allergy Clin Immunol. 2011;127(2):372-81 e371-373.

23. Huang YJ, Nariya S, Harris JM, Lynch SV, Choy DF, Arron JR, Boushey H. The airway microbiome in patients with severe asthma: associations with disease features and severity. J Allergy Clin Immunol. 2015;136(4):874-84.

24. Goleva E, Jackson LP, Harris JK, Robertson CE, Sutherland ER, Hall CF, Good JT Jr, Gelfand EW, Martin RJ, Leung DY. The effects of airway microbiome on corticosteroid responsiveness in asthma. Am J Respir Crit Care Med. 2013;188(10):1193-201.

25. Nathan RA, Sorkness CA, Kosinski M, Schatz M, Li JT, Marcus P, Murray JJ, Pendergraft TB. Development of the asthma control test: a survey for assessing asthma control. J Allergy Clin Immunol. 2004;113(1):59-65.

26. Langille MG, Zaneveld J, Caporaso JG, McDonald D, Knights D, Reyes JA, Clemente JC, Burkepile DE, Vega Thurber RL, Knight R, et al. Predictive functional profiling of microbial communities using 165 rRNA marker gene sequences. Nat Biotechnol. 2013;31(9):814-21.

27. Darzi Y, Falony G, Vieira-Silva S, Raes J. Towards biome-specific analysis of meta-omics data. ISME J. 2016;10(5):1025-8.

28. Montero P, Vilar JA. TSclust: An R package for time series clustering. J Stat Softw. 2014;62(1):1-43.

29. Schatz M, Sorkness CA, Li JT, Marcus P, Murray JJ, Nathan RA, Kosinski M, Pendergraft TB, Jhingran P. Asthma control test: reliability, validity, and responsiveness in patients not previously followed by asthma specialists. J Allergy Clin Immunol. 2006;117(3):549-56.

30. Birzele LT, Depner M, Ege MJ, Engel M, Kublik S, Bernau C, Loss GJ, Genuneit J, Horak E, Schloter M, et al. Environmental and mucosal microbiota and their role in childhood asthma. Allergy. 2017;72(1):109-19.

31. Kirjavainen PV, Karvonen AM, Adams RI, Taubel M, Roponen M, Tuoresmaki P, Loss G, Jayaprakash B, Depner M, Ege MJ, et al. Farm-like indoor microbiota in non-farm homes protects children from asthma development. Nat Med. 2019;25(7):1089-95.

32. Liu H-Y, C-x L, Z-y L, S-y Z, W-y Y, Y-m Y, Y-x L, R-c C, H-w Z, Su J. The Interactions of Airway Bacterial and Fungal Communities in Clinically Stable Asthma. Front Microbiol. 2020;11:1647.

33. Salo PM, Arbes SJ Jr, Sever M, Jaramillo R, Cohn RD, London SJ, Zeldin DC Exposure to Alternaria alternata in US homes is associated with asthma symptoms. J Allergy Clin Immunol. 2006;118(4):892-8.

34. Masaki K, Fukunaga K, Matsusaka M, Kabata H, Tanosaki T, Mochimaru T, Kamatani T, Ohtsuka K, Baba R, Ueda S, et al. Characteristics of severe asthma with fungal sensitization. Ann Allergy Asthma Immunol. 2017;119(3): $253-7$. 
35. Licorish K, Novey HS, Kozak P, Fairshter RD, Wilson AF. Role of Alternaria and Penicillium spores in the pathogenesis of asthma. J Allergy Clin Immunol. 1985;76(6):819-25.

36. Edwards MR, Bartlett NW, Hussell T, Openshaw P, Johnston SL. The microbiology of asthma. Nat Rev Microbiol. 2012;10(7):459-71.

37. Denner DR, Sangwan N, Becker JB, Hogarth DK, Oldham J, Castillo J, Sperling Al, Solway J, Naureckas ET, Gilbert JA, et al. Corticosteroid therapy and airflow obstruction influence the bronchial microbiome, which is distinct from that of bronchoalveolar lavage in asthmatic airways. J Allergy Clin Immunol. 2016;137(5):1398-405 e1393.

38. Zhu J, Thompson CB. Metabolic regulation of cell growth and proliferation. Nat Rev Mol Cell Biol. 2019;20(7):436-50.

39. Stenholm S, Maggio M, Lauretani F, Bandinelli S, Ceda GP, Di lorio A, Giallauria F, Guralnik JM, Ferrucci L. Anabolic and catabolic biomarkers as predictors of muscle strength decline: the InCHIANTI study. Rejuvenation Res. 2010;13(1):3-11.

40. Mouraux S, Bernasconi E, Pattaroni C, Koutsokera A, Aubert JD, Claustre J, Pison C, Royer PJ, Magnan A, Kessler R, et al. Airway microbiota signals anabolic and catabolic remodeling in the transplanted lung. J Allergy Clin Immunol. 2018;141(2):718-29 e717.

41. Caruso M, Holgate ST, Polosa R. Adenosine signalling in airways. Curr Opin Pharmacol. 2006:6(3):251-6.

42. da Rocha LF, de Oliveira AP, Accetturi BG, de Oliveira MI, Domingos HV, de Almeida CD, de Lima WT, Santos AR. Anti-inflammatory effects of inosine in allergic lung inflammation in mice: evidence for the participation of adenosine A2A and a 3 receptors. Purinergic Signal. 2013;9(3):325-36

43. He B, Hoang TK, Wang T, Ferris M, Taylor CM, Tian X, Luo M, Tran DQ, Zhou J, Tatevian N, et al. Resetting microbiota by Lactobacillus reuteri inhibits $T$ reg deficiency-induced autoimmunity via adenosine A2A receptors. J Exp Med. 2017;214(1):107-23.

44. Ostroukhova M, Goplen N, Karim MZ, Michalec L, Guo L, Liang Q, Alam R. The role of low-level lactate production in airway inflammation in asthma. Am J Phys Lung Cell Mol Phys. 2012;302(3):L300-7.

45. Xu YD, Cui JM, Wang Y, Yin LM, Gao CK, Liu YY, Yang YQ. The early asthmatic response is associated with glycolysis, calcium binding and mitochondria activity as revealed by proteomic analysis in rats. Respir Res. 2010;11:107.

46. George F, Daniel C, Thomas M, Singer E, Guilbaud A, Tessier FJ, RevolJunelles AM, Borges F, Foligne B. Occurrence and dynamism of lactic acid Bacteria in distinct ecological niches: a multifaceted functional health perspective. Front Microbiol. 2018;9:2899.

47. de Vries SP, van Hijum SA, Schueler W, Riesbeck K, Hays JP, Hermans PW, Bootsma HJ. Genome analysis of Moraxella catarrhalis strain BBH18, [corrected] a human respiratory tract pathogen. J Bacteriol. 2010;192(14): 3574-83.

48. Bassis CM, Erb-Downward JR, Dickson RP, Freeman CM, Schmidt TM, Young VB, Beck JM, Curtis JL, Huffnagle GB. Analysis of the upper respiratory tract microbiotas as the source of the lung and gastric microbiotas in healthy individuals. mBio. 2015;6(2):e00037.

49. Bisgaard H, Hermansen MN, Buchvald F, Loland L, Halkjaer LB, Bonnelykke K, Brasholt M, Heltberg A, Vissing NH, Thorsen SV, et al. Childhood asthma after bacterial colonization of the airway in neonates. N Engl J Med. 2007; 357(15):1487-95

\section{Publisher's Note}

Springer Nature remains neutral with regard to jurisdictional claims in published maps and institutional affiliations.

Ready to submit your research? Choose BMC and benefit from:
- fast, convenient online submission
- thorough peer review by experienced researchers in your field
- rapid publication on acceptance
- support for research data, including large and complex data types
- gold Open Access which fosters wider collaboration and increased citations
- maximum visibility for your research: over 100M website views per year
At BMC, research is always in progress.
Learn more biomedcentral.com/submissions

